\title{
Correction to: Diagnosis and management of transthyretin familial amyloid polyneuropathy in Japan: red-flag symptom clusters and treatment algorithm
}

\author{
Yoshiki Sekijima ${ }^{1}$, Mitsuharu Ueda ${ }^{2}$, Haruki Koike ${ }^{3}$, Sonoko Misawa ${ }^{4}$, Tomonori Ishii ${ }^{5}$ and Yukio Ando $2^{2^{*}}$
}

\section{Correction to: Orphanet Journal of Rare Diseases (2018) 13:6 https://doi.org/10.1186/s13023-017-0726-x}

The authors would like to rectify the following errors in their published article [1]:

In Fig. 3 of the article (page 7 of the PDF), 'Val30Met' should be omitted from the figure legend, which should instead read: 'Red-flag symptom clusters specific to ATTR-FAP in Japan...'.

Similarly, in Fig. 5 of the article (page 10 of the PDF), 'Val30Met' should be omitted from the figure legend, which should instead read: 'Treatment algorithm specific to ATTR-FAP in Japan....

The authors believe that the applicability of the red-flag symptom clusters and treatment algorithm for this disease should not be limited to the Val30Met mutation and would like to present this with this Correction article.

Furthermore, in Fig. 5 of the article (page 10 of the PDF), the treatment algorithm, Modified body mass index (mBMI) <600, listed as the 2nd bullet under 'Evaluate for conventional LT indications', should be changed to 'Modified body mass index (mBMI) $>600$ '.

This error occurred due to an inadvertent oversight.

Author details

'Department of Medicine (Neurology and Rheumatology), Shinshu University School of Medicine, Matsumoto, Japan. ${ }^{2}$ Department of Neurology, Graduate School of Medical Sciences, Kumamoto University, 1-1-1 Honjo, Chuo-ku, Kumamoto-shi, Kumamoto 860-8556, Japan. ${ }^{3}$ Department of Neurology, Nagoya University Graduate School of Medicine, Nagoya, Japan.

${ }^{4}$ Department of Neurology, Graduate School of Medicine, Chiba University, Chiba, Japan. ${ }^{5}$ Pfizer Japan Inc., Tokyo, Japan.
Received: 9 May 2019 Accepted: 9 May 2019

Published online: 21 May 2019

\section{Reference}

1. Sekijima et al., Diagnosis and management of transthyretin familial amyloid polyneuropathy in Japan: red-flag symptom clusters and treatment algorithm. Orphanet Journal of Rare Diseases (2018) 13:6 https://doi. org/10.1186/s13023-017-0726-x.

* Correspondence: andoy709@kumamoto-u.ac.jp

${ }^{2}$ Department of Neurology, Graduate School of Medical Sciences, Kumamoto

University, 1-1-1 Honjo, Chuo-ku, Kumamoto-shi, Kumamoto 860-8556, Japan

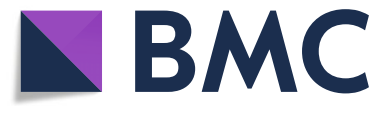

(c) The Author(s). 2019 Open Access This article is distributed under the terms of the Creative Commons Attribution 4.0 International License (http://creativecommons.org/licenses/by/4.0/), which permits unrestricted use, distribution, and reproduction in any medium, provided you give appropriate credit to the original author(s) and the source, provide a link to the Creative Commons license, and indicate if changes were made. The Creative Commons Public Domain Dedication waiver (http://creativecommons.org/publicdomain/zero/1.0/) applies to the data made available in this article, unless otherwise stated. 